\title{
Hemispheric asymmetries in the time course of recognition memory
}

\author{
KARA D. FEDERMEIER and AARON S. BENJAMIN \\ University of Illinois at Urbana-Champaign, Urbana, Illinois
}

\begin{abstract}
Hemispheric specialization has been studied extensively within subfields ranging from perception to language comprehension. However, the study of asymmetries for basic memory functions-an area that holds promise for bridging these low- and high-level cognitive domains-has been sporadic at best. We examined each hemisphere's tendency to retain verbal information over time, using a continuous recognition memory task with lateralized study items and central test probes. We found that the ubiquitous advantage of the left hemisphere for the processing and retention of verbal information is attenuated and perhaps even reversed over long retention intervals. This result is consistent with theories that propose differences in the degree to which the hemispheres maintain veridical versus semantically transformed representations of the input they receive.
\end{abstract}

Despite their anatomical and physiological similarity, the left and right cerebral hemispheres have different information-processing capacities and strengths. These differences range from the nature of the perceptual information that each hemisphere preferentially processes and attends, to the well-known, striking asymmetries for higher cognitive functions, such as language (Hellige, 1993). The different patterns of sensory analysis and consequent response selection suggest that the hemispheres may also fundamentally diverge in the records that they keep of an experience - that is, in their memory representations and functions. However, hemispheric specialization in basic memory tasks remains understudied, particularly in nonpathological populations. This is surprising, since such differences have implications not only for how the two hemispheres remember individual stimuli or events, but also for how each might integrate information over time, as during language comprehension. In this article, therefore, we examine recognition performance as a function of study-test interval for words encoded laterally, testing predictions derived from recent work on language comprehension, as well as from prior studies of memory asymmetries.

The most commonly reported hemispheric asymmetry in memory is a general bias in the tendency to encode verbal, as opposed to nonverbal, information. Studies of patients with unilateral damage to or resection of the an-

We thank Kristen Jackson, Matt Rambert, and Tia Schoth for assistance with data collection. Correspondence concerning this article should be addressed to K. D. Federmeier, Department of Psychology, University of Illinois, Urbana-Champaign, 603 E. Daniel, Champaign, IL, 61820 (e-mail: kfederme@uiuc.edu). terior temporal lobe have reported verbal memory deficits that are more pronounced after left-hemisphere $(\mathrm{LH})$ than after right-hemisphere (RH) lesions, whereas damage to corresponding areas in the RH has, instead, been associated with memory deficits for various kinds of nonverbal information (see, e.g., Falk, Cole, \& Glosser, 2002; Pillon et al., 1999). These results are in agreement with those of behavioral studies in which the visual half-field (VF) presentation technique has been used with brainintact individuals. This technique takes advantage of the fact that information in one half of visual space is initially processed exclusively by early visual cortical areas in the contralateral hemisphere. Although some information may be rapidly relayed across the corpus callosum, this technique leads to preferential processing by the directly stimulated hemisphere, because of the resulting temporal advantage and degradation of information associated with callosal transfer (e.g., Zaidel, 1983). Studies in which VF presentation has been used have uncovered a number of consistent performance differences for stimuli presented to the right visual field (RVF) and the left visual field (LVF) that have been corroborated by evidence from patients with unilateral brain damage or from studies of commissurotomized ("split-brain") patients. In this case, consistent with neuropsychological research, studies in which the VF presentation technique has been used have typically shown an advantage, in terms of response accuracy, speed, or both, for the recognition of verbal material (letters and words) when it is projected to the RVF and, thus, processed initially by the LH (Blanchet, Desgranges, Lechevalier, Eustache, \& Faure, 2001; Coney \& MacDonald, 1988; Jordan, Patching, \& Thomas, 2003; Leehey \& Cahn, 1979). Advantages have also been observed, although with somewhat less consistency, for the 
recognition of various types of nonverbal material when it is projected to the LVF/RH (Blanchet et al., 2001; Dee \& Fontenot, 1973; Leehey \& Cahn, 1979).

The interpretation of these material-specific biases has been somewhat controversial. They have sometimes been taken to imply a functional independence in the memory systems of the two hemispheres, with the LH responsible for verbal encoding and the $\mathrm{RH}$ for nonverbal encoding (e.g., Saykin et al., 1992). However, patients with unilateral damage to either hemisphere can retain both verbal and nonverbal information, and their memory for both types of stimuli is often impaired, relative to brain-intact controls (e.g., Dobbins, Kroll, Tulving, Knight, \& Gazzaniga, 1998). Thus, the hemispheres may be biased but are not fully selective in the type of information that they store. Furthermore, it has generally been difficult to determine the extent to which apparent memory differences might actually be driven by perceptual or attentional asymmetries and/or stimulus presentation parameters that facilitate information extraction from one VF. Advantages for verbal stimuli presented in the RVF/LH, for example, might arise because the LH is more adept than the $\mathrm{RH}$ at reading visual words (Jordan et al., 2003), because of learned attentional biases toward the RVF for word reading in English (e.g., Pollatsek, Bolozky, Well, \& Rayner, 1981), and/or because the beginnings of words (which are closer to the fovea for RVF presentation) tend to be more informative than the endings (e.g., O'Regan, LevySchoen, Pynte, \& Brugaillère, 1984).

There is thus ample evidence for material-related processing asymmetries, although there remains controversy about when and how such biases arise. It is also clear, however, that both hemispheres are likely involved to some degree in memory for all types of stimuli. This raises the question of whether there might be unique ways in which the two hemispheres encode, maintain, and retrieve information about any given stimulus type. One proposal is that the RH stores information fairly veridically, whereas the LH is more likely to "interpret" stimuli and make inferences from them (Metcalfe, Funnell, \& Gazzaniga, 1995). In the domain of verbal memory, such a proposal predicts that the LH is more likely to remember gist (and to mistakenly remember inferred, schemaconsistent information), whereas the RH is more likely to remember the specific stimuli that were encountered (i.e., the form).

Metcalfe et al. (1995) studied recognition memory in a split-brain patient and found results supporting this view. After studying a list of categorically related words, the patient was more successful at rejecting new words from the same category when these were projected to the LVF/ RH. Fabiani, Stadler, and Wessels (2000) also found behavioral and electrophysiological results suggesting that the RH is less susceptible to semantically related lures. However, this LVF/RH advantage is not always obtained (e.g., Ito, 2001), and Westerberg and Marsolek (2003) actually found a greater tendency for the RH to endorse associatively related distractors. A difference in their design was a modality switch from auditory presentation at study to visual presentation at test; to the extent that perceptual factors play a role in veridical recognition, this switch might have affected the pattern of results. These intriguing hints about differences in how material is retained by the two hemispheres clearly indicate a need for more research.

Interestingly, related distinctions have arisen in the literature on asymmetries in word and sentence comprehension. In these studies, participants have been asked to make speeded word/nonword (lexical decision) judgments to lateralized targets presented after a single word or sentence prime. Studies of this type have suggested that while the LH continuously and rapidly builds an integrated meaning representation, the $\mathrm{RH}$, instead, focuses on individual words and low-level relationships between them (e.g., Chiarello, Liu, \& Faust, 2001). Differences have also been postulated in the nature of the semantic information gleaned from individual words, with the RH showing weaker, more diffuse patterns of semantic activation (the course coding hypothesis; Beeman, 1998) that may unfold more slowly over short time scales (e.g., Koivisto, 1997).

Overall, then, both studies of memory and those of language comprehension suggest that the LH may tend to rapidly incorporate new verbal information into an interpreted representation, whereas the $\mathrm{RH}$ attends to and retains more information about individual word stimuli. The idea that the RH maintains encoded words in a more veridical (and perhaps, semantically less specific) format by foregoing the very processes that afford the LH its advantage in language comprehension suggests an additional prediction concerning the effects of lag-namely, that the retention of information about individual words may decrease more rapidly over a delay for the LH than for the RH.

In fact, surprisingly little is known about the time course of memory in the two hemispheres. Some studies have shown VF effect patterns that change form with the duration of the interval between study and test (e.g., Bevilacqua, Capitani, Luzzatti, \& Spinnler, 1979). Coney and MacDonald (1988) specifically examined the effect of study-test lag on recognition, by presenting lateralized study and test words and varying the number of intervening items. At lags greater than one, they observed a consistent response time (RT) advantage for test words presented to the RVF/LH. A significant effect of VF at study was found at only one lag, with faster RTs to words that had been studied and tested in the same VF. Thus, for this experiment, response measures seemed to be driven by the VF of the test stimulus. However, the LH advantage for word reading makes it difficult to determine the extent to which this pattern might have been dominated by perceptual-processing asymmetries, which, in some cases, can mask more subtle effects of memory (Nagae \& Moscovitch, 2002).

To examine hemispheric differences in memory retention under conditions in which confounds from perceptual-decoding differences would be minimized, in the present study, we lateralized the study presentation of 
words but then tested them centrally over a wide range of lags. It is possible that hemispheric transfer is sufficient to give both hemispheres an accurate representation of the lateralized study word; in this case, we should find little or no difference in accuracy or RT to a central target as a function of study VF. If, instead, VF at study biases aspects of the encoding of words and their retention, those effects should be evident at test. The material-specific hypothesis predicts a general LH advantage for recognition across all lags. However, if, as we hypothesize, information about individual words decays more rapidly in the $\mathrm{LH}$ than in the $\mathrm{RH}$, we might expect an attenuation or even a reversal of this $\mathrm{RVF} / \mathrm{LH}$ advantage at the longer study-test intervals.

\section{METHOD}

\section{Participants}

Thirty-six University of Illinois undergraduates participated in the experiment for course credit. Four participants were dropped because they were unable to suppress saccades to the lateralized stimuli. The experimental data were thus derived from a final total of 32 participants ( 16 female), whose mean age was 20 years (range, 18-27). All were monolingual English speakers with no early second language exposure and were right-handed, as assessed by the Edinburgh inventory (Oldfield, 1971). Average laterality quotient was 9 (range, .6-1), where 1 is strongly right-handed and -1 is strongly left-handed.

\section{Materials}

A total of 567 words were selected from the MRC Psycholinguistic Database (Coltheart, 1981). All were singular nouns, four to six letters in length, with high imageability (500-700) and concreteness (500-700) ratings. Word frequency (Francis \& Kučera, 1982) ranged from 2 to 60 . These parameters were chosen because they are most compatible with the known linguistic capabilities of the RH (Baynes \& Eliassen, 1998), thus reducing asymmetries for word processing as much as possible. Words from this baseline set were randomly selected and assigned to experimental conditions to create 16 experimental lists, each consisting of 256 words with intermixed study and test trials. An additional 16 matched lists were created in which the VF of presentation for each study item was reversed. Thus, across participants, identical words appeared in each VF for each condition. Each list consisted of 112 new test words (tested but unstudied) and 144 studied words (lateralized at study and tested once at fixation), intermixed in a continuous recognition memory design. Studied words were divided equally into nine different lag conditions. Lags of 1 (immediate repetition), 2, 3, 5, 7, 10, 20,30 , and 50 were used, with test items from each lag distributed across the experimental run. ${ }^{1}$ Sixteen items were studied and tested in each lag condition, eight in each visual field.

\section{Procedure}

After electrode application (described below), the participants were seated $100 \mathrm{~cm}$ in front of a computer monitor. They held a response button in each hand and were instructed to passively view (with the aim of remembering) words that appeared in the visual periphery. The need for them to maintain central fixation during lateralized word presentation was emphasized. They were instructed to respond to centrally presented words as quickly and accurately as possible with a yes buttonpress if they had previously seen the word at any point in the experiment and a no buttonpress if they had not. The hand used to respond yes was counterbalanced across participants. The participants were given a short practice block (using proper names) to acclimate them to the procedures and allow an assessment of their ability to suppress saccades to the lateralized study items. After the practice trial, the experimental items were presented in a single run lasting approximately $20 \mathrm{~min}$.

Study and test items were intermixed throughout the experimental run. A black fixation cross, presented at the horizontal center and $0.5^{\circ}$ of visual angle below the vertical center on a uniform white background, remained on the screen throughout the experiment. Words were presented in black capital letters in a sans serif font and subtended $2^{\circ}-3^{\circ}$ of horizontal visual angle (depending on word length) and $0.6^{\circ}$ of vertical visual angle. Study items were presented to the LVF or the RVF, with the nearest edge $2^{\circ}$ of visual angle from horizontal center, for a total duration of $200 \mathrm{msec}$, followed by an interstimulus interval of $2,300 \mathrm{msec}$. Test items were presented in the horizontal center of the screen and remained on the screen until the participant responded; they were followed by a 2,500-msec interstimulus interval. Relatively long interstimulus intervals were used in order to avoid visual masking effects that might complicate interpretation of the data.

\section{Eye Movement Monitoring}

Eye movements were continuously monitored during the experiment, using the electro-oculogram signal recorded from a bipolar montage of $\mathrm{Ag} / \mathrm{AgCl}$ electrodes placed on the outer canthus of each eye. Eyeblinks were also monitored, using a second bipolar montage consisting of an electrode placed on the lower orbital ridge of the left eye and on the forehead above the left eye. Electrode impedances were kept below $10 \mathrm{k} \Omega$. The eye signals were amplified 10,000 times, using Sensorium amplifiers with a half-amplitude cutoff band-pass from 0.02 to $100 \mathrm{~Hz}$. The sampling rate was $250 \mathrm{~Hz}$.

Saccades were detected with an automatic peak-finding algorithm, using a threshold set by the experimenter after visual inspection of each participant's data. The study trials with horizontal eye movements during the $200 \mathrm{msec}$ of stimulus presentation were marked, and the corresponding test trials were excluded from further analysis. On average, fewer than $10 \%$ of the experimental trials were lost due to eye movements (range, $0 \%-25 \%$ ) and there was no difference in the tendency to saccade to RVF or LVF words $(F<1)$.

\section{RESULTS}

The participants falsely identified new test words as old $9 \%$ of the time (range, $0 \%-29 \%$ ) and correctly identified old words as old $71 \%$ of the time (range, $52 \%-93 \%$ ). Hit rates were subjected to an omnibus ANOVA on two levels of VF (RVF and LVF) and nine levels of lag (1, 2, $3,5,7,10,20,30$, and 50). There was a main effect of $\mathrm{VF}$ $[F(1,31)=22.13, p<.001]$, with higher overall hit rates for items originally studied in the RVF $(76 \%)$ than for those studied in the LVF (66\%). There was also a main effect of lag $[F(8,248)=32.58, p<.001]$, with hit rates decreasing with increasing lag. VF and lag did not interact $(F=1.05)$. Figure 1 depicts hit rates as a function of lag and VF.

Mean RTs were subjected to an omnibus ANOVA on two levels of VF (RVF and LVF) and nine levels of lag $(1,2,3,5,7,10,20,30$, and 50). Overall RTs did not differ as a function of VF of study $(\mathrm{RVF}=1,006 \mathrm{msec}$; $\mathrm{LVF}=1,007 \mathrm{msec} ; F<1)$. There was a main effect of lag $[F(8,248)=11.50, p<.001]$, with generally longer RTs at longer lags. There was also a significant interaction between VF and lag $[F(8,248)=2.78, p<.01]$. As can be seen in Figure 2, RTs at short lags were shorter to test items that had been studied in the RVF, but RTs at long lags were shorter to test items that had been studied in the LVF. 


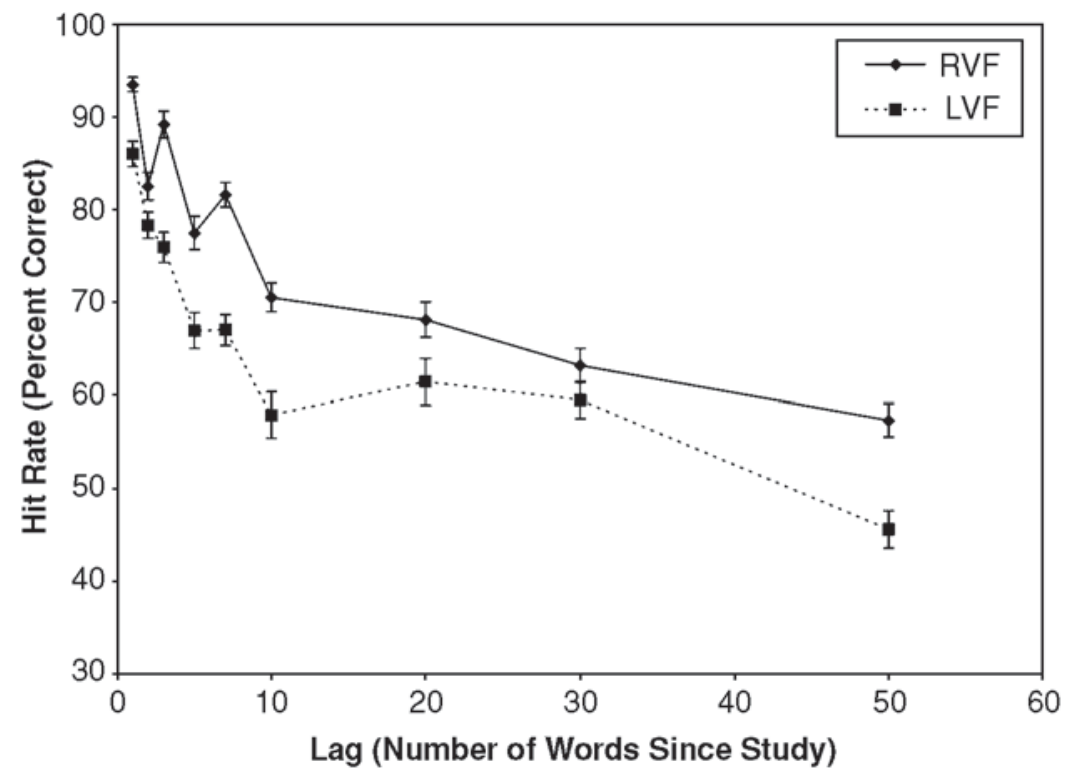

Figure 1. Hit rates (percent correct) for stimuli studied in the right visual field (RVF; diamonds) and the left visual field (LVF; squares) across the nine study-test lags. Error bars give the standard error. Trials in which saccades were detected during study were excluded. The false alarm rate was $9 \%$.

This interaction was tested with two contrasts that allowed us to assess whether the RT difference between the VFs (LVF - RVF) decreased with lag. For the first test, the contrast coefficients were generated as a linear trend matching the unequally spaced lag structure, and this revealed a reliable effect $[F(1,31)=10.98, p<.01]$. For the second contrast, we used a modification of the
Abelson and Tukey (1963) "efficient" test for detection of a monotone trend by quadrupling the outermost and doubling the second outermost coefficients of a linear trend (Keppel \& Wickens, 2004); this also revealed a reliable effect $[F(1,31)=4.92, p<.05]$. Indeed, as can be seen in Figure 2, the difference between the two VFs even changes sign across the lag spectrum: At the four short-

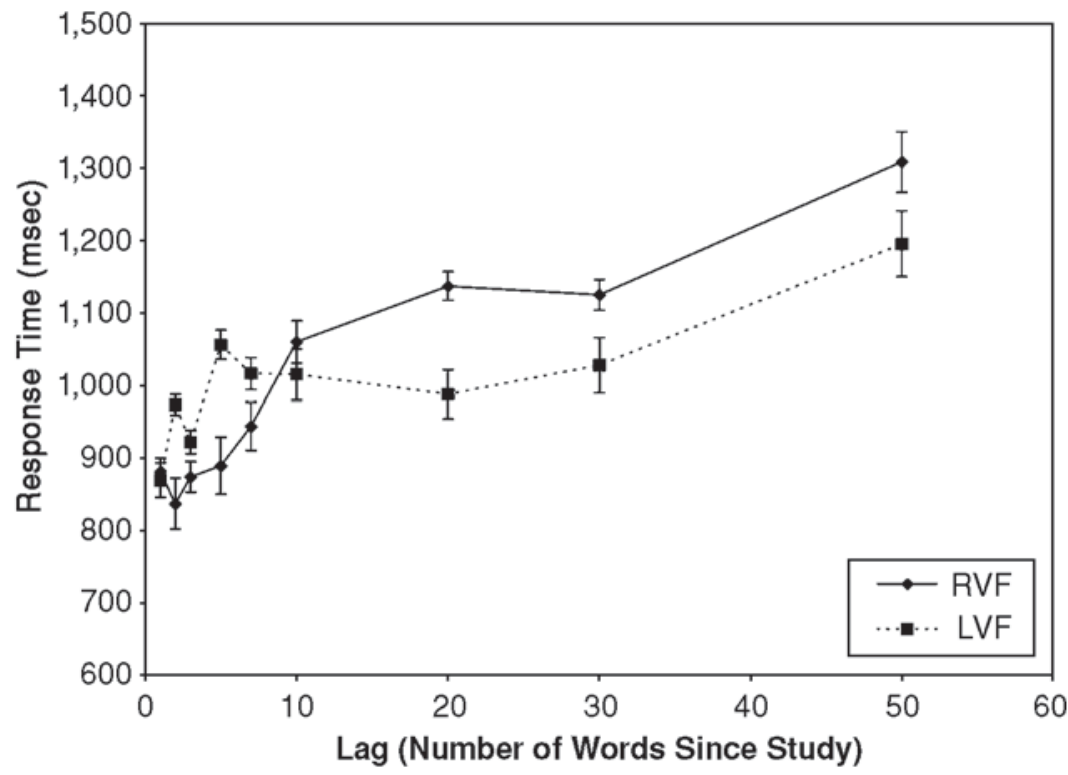

Figure 2. Response times (in milliseconds) for hits to stimuli studied in the right visual field (RVF; diamonds) and the left visual field (LVF; squares) across the nine study-test lags. Error bars give the standard error. Trials in which saccades were detected during study were excluded. 
est lags $(1,2,3$, and 5), responses were faster for words studied in the RVF $(877 \mathrm{msec})$ than for those studied in the LVF [947 msec; $F(1,31)=5.61, p<.05]$, whereas at the four longest lags $(10,20,30$, and 50$)$, responses were faster to words studied in the LVF $(1,051 \mathrm{msec})$ than for those studied in the RVF $[1,154 \mathrm{msec} ; F(1,31)=4.57$, $p<.05] .^{2}$

\section{DISCUSSION}

The goal of this study was to examine whether hemispheric differences in memory for words change over time. In agreement with previous results (e.g., Blanchet et al., 2001; Leehey \& Cahn, 1979), there was a response advantage (higher hit rates and shorter RTs) for RVFstudied words even at Lag 1, where memory demands were minimal. As was outlined in the introduction, this difference was likely due to perceptual-, attentional-, and presentation-related factors that favored word reading (and thus encoding) for RVF items. The question, then, is whether this baseline RVF advantage interacts with lag, suggesting the influence of memory factors that operate differently in the two hemispheres.

There were no VF $\times$ lag interactions in the hit rates, suggesting that the decreased quality and/or quantity of information gleaned from the LVF study words resulted in decreased accuracy in the recognition of those words on second presentation, independent of memory-related factors. However, recognition RTs did reveal lateralized memory differences. At short lags, beginning with Lag 1, responses were faster to words studied in the RVF/LH. This result is consistent with previous studies in which RTs to laterally presented verbal material were measured (e.g., Coney \& MacDonald, 1988). However, this advantage decreased with increasing lag and, at the longer study-test intervals, actually reversed, with faster responses to words that had been studied in the LVF/RH. This pattern is quite striking, given that LH advantages are almost always observed for verbal material (and were present in this data set for immediate repetition). There are several reasons to believe that this RT reversal at the long lags cannot be explained as a developing speed-accuracy trade-off for the LVF-studied items. VF-related accuracy differences did not change over lag (and at several of the longer lags, were numerically smaller than average). Furthermore, there were no VF-based RT differences for misses, showing that the participants were not generically responding more quickly to LVF-studied items tested at long lags. Instead, it seems that the recognition advantage for the $\mathrm{LH}$ was attenuated and even reversed at long lags.

One way of conceptualizing this result is that the RH's performance decreased more gradually with lag than did the LH's, resulting in a reversal of the relative ordering of RTs in favor of the verbally "weaker" hemisphere at the longest lags. This asymmetry in the time course of memory is consistent with the hypothesis that the two hemispheres employ different strategies for the processing of verbal information. For example, if the $\mathrm{LH}$, as evi- denced by its superior language capabilities, tends to rapidly transform verbal information into integrated, higher order representations (extracting gist, using top-down information, and building sentence- and discourse-level representations when possible), it may also tend to more rapidly lose information about specific characteristics of individual stimuli. In contrast, it has been argued that the RH's processing is biased toward individual words (Chiarello et al., 2001) and form-specific aspects of visual stimuli (Marsolek, 1999). This stimulus-specific information may afford items studied with RH-biased strategies a memory advantage under certain circumstances-for example, when verbatim word memory is necessary after relatively long time intervals. Alternatively, it is possible that more diffuse semantic encoding by the RH (e.g., Beeman, 1998) provides a broader base of retrieval cues that can aid in the recognition of a word whose memory representation has been weakened by decay or interference, as across longer lags.

Overall, then, the results suggest that there are important hemispheric differences in the time course with which verbal information is retained, with faster changes for at least some parameters in the LH, as compared with the RH. In turn, this asymmetry in the tendency to retain different kinds of information about words over time may relate to various language comprehension asymmetries, including the tendency to show word-level priming effects in sentences (e.g., Faust, Babkoff, \& Kravetz, 1995), the capacity to use word-specific information to revise inferences and/or reanalyze context information (e.g., Beeman, 1993), and the ability to integrate information over larger discourse contexts more generally (e.g., Delis, Wapner, Gardner, \& Moses, 1983). A better understanding of the temporal course of memory in the two hemispheres thus holds the promise of providing links between asymmetries at various levels of processing and, more generally, provides constraints on the kind of neurobiological mechanisms that may underlie functional differences between the two cerebral hemispheres.

\section{REFERENCES}

Abelson, R., \& Tukey, J. (1963). Efficient utilization of non-numerical information in quantitative analysis: General theory and the case of simple order. Annals of Mathematical Statistics, 34, 1347-1369.

Baynes, K., \& Eliassen, J. C. (1998). The visual lexicon: Its access and organization in commissurotomy patients. In M. Beeman \& C. Chiarello (Eds.), Right hemisphere language comprehension: Perspectives from cognitive neuroscience (pp. 79-104). Mahwah, NJ: Erlbaum.

BeEMAN, M. (1993). Semantic processing in the right hemisphere may contribute to drawing inferences from discourse. Brain \& Language, 44, 80-120.

Beeman, M. (1998). Coarse semantic coding and discourse comprehension. In M. Beeman \& C. Chiarello (Eds.), Right hemisphere language comprehension: Perspectives from cognitive neuroscience (pp. 255-284). Mahwah, NJ: Erlbaum.

Bevilacqua, L., Capitani, E., Luzzatti, C., \& Spinnler, H. R. (1979). Does the hemisphere stimulated play a specific role in delayed recognition of complex abstract patterns? A tachistoscopic study. Neuropsychologia, 17, 93-97.

Blanchet, S., Desgranges, B., Lechevalier, B., Eustache, F., 
\& FAURE, S. (2001). New questions on the hemispheric encoding/ retrieval asymmetry (HERA) model assessed by divided visual-field tachistoscopy in normal subjects. Neuropsychologia, 39, 502-509.

Chiarello, C., Liu, S., \& Faust, M. (2001). Bihemispheric sensitivity to sentence anomaly. Neuropsychologia, 39, 1451-1463.

Coltheart, M. (1981). The MRC psycholinguistic database. Quarterly Journal of Experimental Psychology, 33A, 497-505.

Coney, J., \& MacDonald, S. (1988). The effect of retention interval upon hemispheric processes in recognition memory. Neuropsychologia, 26, 287-295.

Dee, H. L., \& Fontenot, D. J. (1973). Cerebral dominance and lateral differences in perception and memory. Neuropsychologia, 11, 167173.

Delis, D. C., Wapner, W., Gardner, H., \& Moses, J. A. (1983). The contribution of the right hemisphere to the organization of paragraphs. Cortex, 19, 43-50.

Dobbins, I. G., Kroll, N. E. A., Tulving, E., Knight, R. T., \& GaZZANIGA, M. S. (1998). Unilateral medial temporal lobe memory impairment: Type deficit, function deficit, or both? Neuropsychologia, 36, 115-127.

Fabiani, M., Stadler, M. A., \& Wessels, P. M. (2000). True but not false memories produce a sensory signature in human lateralized brain potentials. Journal of Cognitive Neuroscience, 12, 941-949.

Falk, M. C., Cole, L. C., \& Glosser, G. (2002). Pseudoword and real word memory in unilateral temporal lobe epilepsy. Journal of Clinical \& Experimental Neuropsychology, 24, 327-334.

Faust, M., Babkoff, H., \& Kravetz, S. (1995). Linguistic processes in the two cerebral hemispheres: Implications for modularity vs interactionism. Journal of Clinical \& Experimental Neuropsychology, 17, 171-192.

Francis, W. N., \& KučERA, H. (1982). Frequency analysis of English usage: Lexicon and grammar. Boston: Houghton Mifflin.

Hellige, J. B. (1993). Hemispheric asymmetry: What's right and what's left. Cambridge, MA: Harvard University Press.

Iто, Y. (2001). Hemispheric asymmetry in the induction of false memories. Laterality, 6, 337-346.

Jordan, T. R., Patching, G. R., \& Thomas, S. M. (2003). Assessing the role of hemispheric specialisation, serial-position processing, and retinal eccentricity in lateralised word recognition. Cognitive Neuropsychology, 20, 49-71.

KePPEL, G., \& Wickens, T. D. (2004). Design and analysis: A researcher's handbook (4th ed.). Upper Saddle River, NJ: Prentice Hall.

KoIvisto, M. (1997). Time course of semantic activation in the cerebral hemispheres. Neuropsychologia, 35, 497-504.

LEEHEY, S. C., \& CAHN, A. (1979). Lateral asymmetries in the recognition of words, familiar faces and unfamiliar faces. Neuropsychologia, 17, 619-628.
MarsoleK, C. J. (1999). Dissociable neural subsystems underlie abstract and specific object recognition. Psychological Science, 10, 111-118.

Metcalfe, J., Funnell, M., \& Gazzaniga, M. S. (1995). Righthemisphere memory superiority: Studies of a split-brain patient. Psychological Science, 6, 157-164

Nagae, S., \& Moscovitch, M. (2002). Cerebral hemispheric differences in memory of emotional and nonemotional words in normal individuals. Neuropsychologia, 40, 1601-1607.

OLDFIELD, R. C. (1971). The assessment and analysis of handedness: The Edinburgh inventory. Neuropsychologia, 9, 97-113.

O’Regan, J. K., Levy-Schoen, A., Pynte, J., \& Brugaillè̀e, B. (1984). Convenient fixation location within isolated words of different length and structure. Journal of Experimental Psychology: Human Perception \& Performance, 10, 250-257.

Pillon, B., Bazin, B., Deweer, B., Ehrle, N., Baulac, M., \& Dubois, B. (1999). Specificity of memory deficits after right or left temporal lobectomy. Cortex, 35, 561-571.

Pollatsek, A., Bolozky, S., Well, A. D., \& Rayner, K. (1981). Asymmetries in the perceptual span for Israeli readers. Brain \& Language, 14, 174-180.

Saykin, A. J., Robinson, L. J., Stafiniak, P., Kester, D. B., Gur, R. C., O'Connor, M. J., \& Sperling, M. R. (1992). Neuropsychological changes after anterior temporal lobectomy: Acute effects on memory, language, and music. In T. L. Bennett (Ed.), The neuropsychology of epilepsy (pp. 263-290). New York: Plenum.

Westerberg, C. E., \& Marsolek, C. J. (2003). Hemisphere asymmetries in memory processes as measured in a false recognition paradigm. Cortex, 39, 627-642.

ZAIDEL, E. (1983). Disconnection syndrome as a model for laterality effects in the normal brain. In J. B. Hellige (Ed.), Cerebral hemisphere asymmetry: Method, theory, and application (pp. 95-151). New York: Praeger.

\section{NOTES}

1. Mean list position: new, 188; Lag 1, 176; Lag 2, 209; Lag 3, 205; Lag 5, 197; Lag 7, 228; Lag 10, 248; Lag 20, 232; Lag 30, 205; Lag 50, 212 .

2. The same analysis performed on the miss data (for the 28 subjects who had misses at short lags) revealed no effect of VF at either short or long lags [short lag RVF, 1,441 msec; short lag LVF, 1,346 msec; long lag RVF, $1,294 \mathrm{msec}$; long lag LVF, $1,303 \mathrm{msec}$; main effect of VF, $F(1,27)=$ $1.24, p=.28 ; \mathrm{VF} \times$ lag interaction, $F(1,27)=1.05, p=.31]$.

(Manuscript received August 26, 2004; revision accepted for publication March 24, 2005.) 